International Journal of English Literature and Social Sciences
Vol-6, Issue-4; Jul-Aug, 2021

Peer-Reviewed Journal

\title{
The Nature of Evil in William Golding's Lord of the Flies and Manik Bandapaddhaya's Padma Nadir Majhi: An Analysis
}

\author{
Md. Atiqur Rahman, Farhana Yeasmin
}

Student, MA in English, Department of English, Jashore University of Science and Technology, Jashore-7408, Bangladesh Assistant Professor, Department of English, Jashore University of Science and Technology, Jashore-7408, Bangladesh.

Received: 10 Jul 2021; Received in revised form: 05 Aug 2021; Accepted: 12 Aug 2021; Available online: 19 Aug 2021 (C2021 The Author(s). Published by Infogain Publication. This is an open access article under the CC BY license (https://creativecommons.org/licenses/by/4.0/).

\begin{abstract}
This paper explores the nature of evil discussed in Lord of the Flies by William Golding and Padma Nadir Majhi by Manik Bandapaddhaya. In both of the novels, evil is a common phenomenon though the presentations may be different in some cases. In Lord of the Flies, the evil, solely innate in nature, is presented through the children who have turned into savages with the progress of the story. On the island, they do unlawful activities and behave in a way as if they were part of an uncivilized society. On the other hand, in Padma Nadir Majhi evil is presented in the forms of carnal desire and societal exploitation. Many of the characters of the novel are involved in extramarital affairs to fulfill their carnal desire and in this way, they commit evil deeds. Another form of evil depicted in Padma Nadir Majhi is the evil created by the socioeconomic system in society. It is important to note that no matter what a person's age, status, culture, or gender is, he/she possesses evil in his/her character-evil exists everywhere in multidimensional forms and natures. This paper will problematize this issue.
\end{abstract}

Keywords - carnal desire, evil, innate, nature, socio-economic system.

\section{INTRODUCTION}

Evil plays a significant role in human life as well as in literature. Regarding evil William Golding (1983) states, "I must say that anyone who moved through those years without understanding that man produces evil as a bee produces honey, must have been blind or wrong in the head." Lord of the Flies (1954) deals with the nature of evil inherent in human beings. It describes the life and behaviour of a group of boys who find themselves isolated on an island somewhere in the Pacific Ocean and then do evil deeds. Here evil indicates savagery, mercilessness, destruction, bestiality, and brutality of a man lurking below the surface of civilization. In the novel, the boys have turned themselves into savages though they were innocent at the beginning. What makes them evil is the main issue that Golding has exposed. The novelist also portrays the causes and consequences. On the other hand, Padma Nadir Majhi
(1936), a Bengali novel, depicts the life, behaviour, and daily activities of a group of fishermen living in Ketupur located on the bank of the river Padma. The novel, like Lord of the Flies, brings out the inscriptions of evil in human beings. The characters (except one or two) of the novel do evil deeds: some step on an evil path to fulfill their unethical sexual desire, while some use it for attaining social status. Kuber, the protagonist, and Kopila, another central character of the novel, commit evil deeds by fulfilling their carnal desire exceeding the ethics exercised in society. Hossain Mia, a crucial character, is also prone to evil and exploits the fishermen. In these two novels, evil is one of the prime determiners that govern the lives and destinies of the characters. This paper will problematize the nature of evil presented in the texts. 


\section{THE NATURE OF EVIL IN LORD OF THE FLIES}

In Lord of the Flies, the young schoolboys commit evil deeds and turn themselves into savages on the island. The novel launches a story filled with symbolic meaning. It deals with the nature of human society as well as the clash that happens within a human-the battle between good and adverse. The island at the beginning represents a Hobbesian situation but gradually turns into a chaotic or evil one. The young schoolboys establish a society and form a government there. They are supposed to behave rationally and live peacefully but they do not behave so. They start their journey towards evil. Their humanity and innocence are lost, inwardly they transform into beasts-uncontrollable, dangerous. For their evil nature, especially for the activity of Jack and his choir, the boys lose the Garden of Eden where they could play and live happily. It echoes Milton's Paradise Lost where Adam and Eve lose the Garden of Eden for the allurement of Satan. The evil committed by the boys gradually becomes stronger and more self-assertive; especially when Jack and his choir become more ambitious and more defiant of Ralph's authority.

The first sign of evil is noticed in the activity of Jack Merridew, a Machiavellian villain, and his choir on the seemingly tranquil island. Jack and his choir are belligerent and aggressive. Jack is the symbol of true evil who expresses a satanic impression. He is like the witches in Macbeth who know "fair is foul, foul is fair" (Shakespeare, 1606 , p. 1) or like Hitler who believes in the holocaust. He commands his choir like a tyrant without allowing room for neither talk nor dissent. The evil nature of Jack is explicit when the boys vote Ralph, who represents a democratic ethos, to make him chief. Jack makes instant conflict and wants to achieve the role of a leader automatically defeating Ralph who achieves it by vote.

Ralph, a democratic leader and chief of the conch, asks for the opinions of Jack on different issues. While Ralph exercises democracy and justice, Jack misuses these as means for punishing and controlling Ralph and his followers. It reflects his authoritarian spirit and tendency towards evil and violence. He remains more fascinated in hunting and causing disorder and pain than contributing or creating anything of use. Jack believes himself superior to the boys. Golding points out that there is something extremely dangerous in Jack's obsession. He represents the negative, brutal, and darker urges of mankind while Ralph represents the opposite. Their differences in opinion, belief, and behaviour are growing stronger and a clash takes place in consequence.

The conflict among the boys, especially between Ralph and Jack, gradually reaches its peak. Jack remains a destructive and aggressive force throughout the story. He continues to threaten Piggy and manipulates the young boys' fear of the possible beast. He promotes anarchy and leads them to disorder. Jack does not care for rules-

“The rules!” shouted Ralph. "You're breaking the rules!"

"Who cares?"

Ralph summoned his wits.

"Because the rules are the only thing we've got!"

But Jack was shouting against him.

"Bollocks to the rules! We're strong-we hunt! If there's a beast, we'll

hunt it down! We'll close in and beat and beat and beat-!" (Golding, 1954, p. 100)

The struggle between Jack and Ralph becomes more intense as Ralph's goodness continually reminds Jack of his misdeeds. While Ralph concentrates on escaping and survival, Jack remains obsessed with playing and hunting. After Jack takes hold of the control over the boys, most of them are influenced by him and support him. He exercises evil and makes the boys evil but Ralph remains the same. However, Ralph also has to follow Jack's command. The boys become dirty and uncivilized under the leadership of Jack. So, civilization is not a corrupter; man corrupts civilization which is evident in Jack and his choirs.

Jack continues to descend to savagery and violence. He denies the rules usually exercised in a civilized society; he only believes in power over others. Here Jack is a dictator who rules by force and threat; none can dare to speak. Ralph represents a liberal democrat who governed the boys by choice, respect, and mutual understanding where each could speak and was respected. The struggle between Jack and Ralph occurs again when Jack orders the boys to join in the circle. Ralph tries to convince the boys, but Jack is more powerful now. He leads almost all the boys and urges them to dance, "Do our dance! Come on! Dance!" (Golding, 1954, p. 170). Finding no other way, Ralph and Piggy also join the circle. By joining the savage act, they also surrender to savagery.

There is a constant dichotomy between good Ralph and evil Jack as Ralph still holds a strong moral sensibility. Piggy also has a strong sense of morality. They are ashamed of the murder done by Jack and his boys even though they were not directly involved in the murder. On the contrary, Jack loses all sense of morality and he likes anarchy, savagery, barbarities, brutalities, etc. He no longer resides in civilized culture rather in a wild atmosphere, he turns into the wild inside. While Ralph and Piggy try to lead their life in line with the norms of the civilized worlds, Jack and his hunters feel irritated noticing such endeavor. The evil succumbs the boys, they become more and more brutal. Maurice and Roger gladly accompany Jack when he raids 
Ralph's camp and grabs Piggy's spectacles, though they know that Piggy would be rendered almost blind without his spectacles.

The boys, under Jack's leadership now symbolize the collapsed nature of man-the cruelty of man. Jack has metamorphosed into a real devil, with Roger and Maurice as his chief lieutenants. When Piggy and Ralph go to Jack to get the glasses back, the greatest tragedy takes place. A confrontation occurs between the civilized Ralph and his fellow and the animalistic Jack and his choir. At one point of their confrontation, Piggy criticizes their animalistic attitudes asking them whether it is better to live prudently or sensibly abiding by the rules and standards of behaviour or to live in a state of anarchy, "Which is better-to be a peak of painted niggers like you are, or to be sensible like Ralph is? . . . Which is better, law and rescue, or hunting and breaking things up?" (Golding, 1954, p. 205). The boys pay no heed to him as if it was not a matter to bother about. While Piggy talks to them about their evil/savage nature, Roger throws a rock to kill Piggy. The rock strikes Piggy and his head splits. Ralph is also attacked with a spear and is wounded. The twins have already been seized and tied up under Jack's order. Ralph flees from the spot to save his life. Not only Jack but all his savages have now become bloodthirsty and determined to murder Ralph.

We find a brilliant portrayal of the evil in man in the final chapter. The boys, except Ralph and his fellows, are now savages losing their moral or rational sensibility. The protagonist, Ralph, cannot defend the boys through any sense of morality or justice. He realizes that the experience on the island is an end of innocence as the boys choose wildlife over rationality and awareness. Significantly, Jack's hatred for Ralph has become so fierce that only the murder of Ralph would satisfy him. He tries to kill Ralph; however, Ralph luckily manages to escape from Jack and his choir. Human nature shows itself at its worse in these boys who are crueler than the beasts.

\section{THE NATURE OF EVIL IN PADMA NADIR MAJHI}

Manik Bandapaddhaya's Padma Nadir Majhi is a stark example of evil in humans. The evil presented here is the exposition of the evil that exists in the human psyche that directly influences external behaviour. The characters are prone to go beyond the conventional boundaries of goodness and do evil deeds. On the one hand, the central characters like Kuber, Kopila, Rashu, Enayet, and Bashir's wife try to fulfill their unethical sexual desire, and Hossain Mia, Nokul Das, and Shital Ghosh exploit the poor on the other.

Manik Bandapaddhaya explains unethical sexual desire in Padma Nadir Majhi. Influenced by Freudian psychology, he discusses human sexual appetite for which people are running around. This hunger lies at the root of human activities. By describing the remote public life of Ketupur, Chardanga, Aminbari, Akurtakur, and Moinadip, Manik shows the evil forces of carnal in the characters' lives. A strong attraction to sexuality is noticed not only in the characters of Kuber and Kopila but also in the characters of Rashu, Enayet, and Bashir's wife.

Kuber and Kopila feel sexual desire for each other. Their intimacy develops when Kuber brings Kopila to his shabby hut during the natural calamity. Their relationship is undoubtedly unethical because they have their families; wife/husband, children, and other members. Kopila provokes Kuber's primitive desire through her skills and tricks. Though Kuber does not notice the signals of Kopila at the beginning, gradually he comes to realize that Kopila has cast unavoidable spells on him from which he cannot escape or he does not want to escape. Kopila becomes essential for him for she offers him the care that he has never received even from his wife in his life. After working all night on the Padma, when Kuber comes home and gets everything without asking, his attraction towards Kopila increases naturally.

Kopila also fulfills her carnal desire through Kuber. She knows she can attract Kuber by friskiness. After Gopi is admitted to Aminbari Hospital, everyone returns to Ketupur, however, Kopila remains with Kuber. She is reluctant to miss the opportunity to spend time with Kuber. She is not even worried about the stigma for this. Eventually, at Kopila's persuasion, Kuber rents a room of a hotel for six annas. Though Kuber tries to keep him separate from Kopila, Kopila remains fixed on spending the night together and finally does so. In the conversation between Kuber and Kopila, we see that Kopila insists Kuber sleep beside her that night on the same mat:

\section{কুবের উঠিয়া দাঁড়ায় বলে, ‘শো কপিলা’। \\ 'তুমি কই যাও' \\ ‘উই পাটিতে শুই গিয়া আমি, যামু কই?' ... \\ 'ডরামু মাঝি'। (বন্দ্যোপাধ্যায়, ১৯৩৬, পৃ. ১০৬)}

(Kuber stood up and said, "Kopila, sleep."

"Where are you going?"

"I am sleeping on that mat, where will I go?"

"I will be scared") [Our translation]

The intimacy between Kuber and Kopila reaches to that level that even separation for a single day is unendurable to Kuber. After two days when Kuber goes to see his daughter at Aminbari Hospital, Kopila expresses her desire to go with him. But due to Mala's reluctance and Kuber's cowardice, she cannot accompany him. The next 
day, after returning from the hospital, Kuber sees Kopila's husband Shayama Das in his house who has come to take Kopila with him as his second wife has died. Kuber is jealous of him and becomes arrogant, even does not talk to Kopila all day. Kopila leaves Kuber's house and Kuber cannot concentrate on his work after her departure. Kopila's absence generates a void in his heart. To meet Kopila, he comes to Kopila's house one morning. Kopila easily understands his real purpose and mocks him. So, despite Shayama Das's request, Kuber refuses to stay there. He curses Kopila for her indifference to him and angrily leaves the house, "গরু ছাগল ভাবস আমারে তুই, খেলা করস আমার লগে? তরে চিনে গেলাম কপিলা, পরনাম কইরা গেলাম তরে” (বন্দ্যোপাধ্যায়, ১৯৩৬, পৃ. ১৪৫). ("Do you think me a cow or a goat, do you play with me? I have recognized you, Kopila, I curse you.”) [Our translation]

However, Kopila's irresistible attraction occupies Kuber so much that he cannot forget Kopila. When he knows that Kopila has come to Chardanga for a few days, Kuber becomes desperate to go and goes to meet Kopila. At one point in the exchange of greetings, he asks Kopila whether she is ill. In response to the question, Kopila frankly says that she is tormented with a dilemma for she gets tired of thinking of Kuber but finds no way out to be united. She cannot deny that Kuber is Mala's husband, the only earning member of the family. She also cannot reject that she wants Kuber solely. Eventually, she responds to the call of her heart. At the end of the novel, when Kuber is charged with stealing, Kopila encourages him to go for Moinadip and accompany him. Kuber also thinks that Kopila should go with him. Thus, both Kuber and Kopila ignoring their past or families go for Moinadip to settle there:

ঘাটের খানিক তফাতে হোসেনের প্রকাণ্ড নৌকাটি নোঙর করা ছিল। একজন মাঝি ঘুমাইয়া ছিল নৌকায়। তাকে ডাকিয়া তুলিলে সে নৌকা তীরে ভিড়াইল। কুবের নীরবে নৌকায় উঠিয়া গেল। সঙ্গে গেল কপিলা। ছইয়ের মধ্যে গিয়া সে বসিল। কুবেরকে ডাকিয়া বলিল, ‘আমারে নিবা মাঝি লগে?' হ, কপিলা চলুক সঙ্গে।একা অত দূরে কুবের পাড়ি দিতে পারবে না। (বন্দ্যোপাধ্যায়, ১৯৩৬, পৃ. ১৭০)

(Hossain's gigantic boat was parked at a short distance from the wharf. A boatman was sleeping in the boat. When he was called, he brought the boat ashore. Kuber silently got into the boat. Kopila went with Kuber. She got into the boat and sat. She called Kuber and said, "Will you take me with you?" Yes, Kopila can go with him. Kuber will not be able to travel that far alone.) [Our translation]

Carnal desire also dominates Rashu's life. When he comes back to Ketupur, abandoning the wife and children on Moinadip, the vitality in him seems exhausted. But people never stop. After returning to Ketupur and being fascinated by Gopi, Rashu starts dreaming of a new life. He stands on the side of the deserted road to see Gopi. Sometimes he gives her gift items. Behind all these activities, a strong desire for Gopi is hidden. He maintains a good relationship with Kuber only for getting Gopi. When Kuber arranges Gopi's marriage to Banku, Rashu publicly threatens him by saying that he will take revenge. He hides Pitam Majhi's, his maternal uncle, stolen money in Kuber's house. Fearing the police, Kuber decides to move to Moinadip on the advice of Hossain Mia. So Rashu's sexual desire has a significant impact on his activities and the storyline of this novel.

Enayet and Bashir's young wife's love on Moinadip is also based on sexual desire. Bashir's old age and his sexual weakness make his young wife unhappy. Dissatisfied with sexual life, the young bride provokes Enayet. One day when everyone goes to work, Enayet enters Bashir's room at noon. Although Bashir's wife starts shouting when she sees Enayet, she has the desire for him, otherwise, she wouldn't go to Enayet to feed him quietly at night when he is being punished for entering into another bride's room. Perhaps, she shouted at because her neighbor could see their intimacy in broad daylight. Hossain Mia plays a momentous role in unethical sexual activities. All he wants is to have a community on his island. On Moinadip, he builds up an open place where the citizens can exercise their sexual activities freely, "Moinadip seems to be a free land of sexual attraction beyond the question of familystructure, social bond, religious norms, and moral code of life" (Hossain, 2018, p. 24). Thus, we see not only Kuber and Kopila, but also Rashu, Enayet and Bashir's wife have their illicit sexual thirst and it plays a vital role in defining the outcome of this novel.

We can also see the nature of evil through the lens of societal exploitation. Manik Bandapaddhaya has exposed the social picture of Ketupur in Padma Nadir Majhi. Here, we see that the rich do evil deeds to exploit, and the downtrodden fall victim to it. The characters like Hossain Mia, Dhananjoy, Shital Ghosh, and Chalan babu Kedarnath are deeply interested in money-making. Hossain Mia exploits the fishermen covertly. He tactfully captures the inhabitants of Ketupur and sends them to Moinadip to fulfill his desires. When he came to Ketupur, he was poor. After that, he becomes the most powerful man in the clan. However, the source of his power and money is unknown to 
the inhabitants. He is a mysterious character who sends several families and people of Ketupur to Moinadip to form a community. Though Moinadip is not habitable, the people have to stay there because they all are afraid of Hossain Mia. The inhabitants of Ketupur are like the puppets on the hand of Hossain. He exploits the fishermen through his superior economic position. He fulfills their basic needs that is why the fishermen of Ketupur are unable to oppose him. Kuber also gets help from Hossain Mia several times for his livelihood. When Hossain Mia offers Kuber to work in his boat, he takes the work because he has to survive. Though Kuber knows, "হোসেন মিয়ার উপকার গ্রহণ করিলে শেষ পর্যন্ত মঙ্গল নাই, কুবের তাহা ভাল করিয়াই জানে, কোন স্বার্থ সাধিবার জন্য কবে কাহাকে হোসেন মিয়ার প্রয়োজন হয় তাহাই শুধু কেহ জানিতে পারে না . . ." (বন্দ্যোপাধ্যায়, ১৯৩৬, পৃ. ১১৪) (Kuber knows well that no good will come out in the end in accepting Hossain Mia's help. No one can tell whom Hossain Mia will call upon to serve what purpose ...) [Our translation]. Hossain encourages the fishermen to give up their profession to fulfill his demands. Influenced by Hossain Mia, the fishermen go for Moinadip to assist him in establishing his long-cherished settlement.

Dhananjoy is another evil character who, like Hossain Mia, exploits the fishermen of Ketupur. He is the owner of the boat and the net. Kuber and Gonesh work with him but get less money than their rights. They catch a lot of fishes but Dhananjoy hides its number. Kuber understands Dhananjoy's cunningness but cannot protest. He has to accept this cheating and injustice without any protest. This exploitation is continuing generation after generation. To describe the poor condition of the fishermen of Ketupur, the novelist at one point utters, "ঈশ্বর থাকেন ওই গ্রামে, ভদ্র পল্লীতে। এখানে তাঁহাকে খুঁজিয়া পাওয়া যাইবে না।", ববন্দ্যোপাধ্যায়, ১৯৩৬, পৃ. ১৪০) (God lives in that village, in that gentle countryside. He would not be found here) [Our translation]. Shital Ghosh and Chalan babu Kedarnath are also symbols of evil. They also exploit the fishermen in different ways like Hossain Mia and Dhananjoy. Shital takes fish from Kuber but sometimes gives him no money. The conversation between Kuber and Shital Ghosh shows the exploitation, “'পয়সা কাইল দিমু কুবের। 'রন শেতল বাবু, অমন তরা কইরা যাইবেন না, দামটা দ্যান দেখি। 'কাইল দিমু কইলাম যে?' 'অই, অখন দ্যান। খামুনা? পোলাগো খাওয়ামুনা?’” (বন্দ্যোপাধ্যায়, ১৯৩৬, পৃ. ৩৭) ("'I will pay tomorrow Kuber.' 'Wait Shital Babu, don't leave so soon, go after payment.' 'I told you that I would pay you tomorrow?' 'Give it now, don't we eat? Don't I feed my boys?"') [Our translation]. The boatmen in Ketupur work hard for their livelihood but cannot change their lots anymore. They never get a fair price. If they get more fish, they have to subscribe five fishes to Chalan Babu Kedarnath. So, they are cheated on in every possible way by the ruling class people, and it seems to be their born-curse. According to the novelist, "এমনভাবে জেলেদের বঞ্চিত করিবার অধিকারটা সকলে তাই প্রথার মত, সামাজিক ও ধর্ম সম্পর্কীয় দশটা নিয়মের মত, অসংকোচে গ্রহণ করিয়াছেন।" (বন্দ্যোপাধ্যায়, ১৯৩৬, পৃ. ৩৮) (The right to deprive fishermen in this way has been unhesitatingly accepted by all, as a matter of custom, like the other social and religious rules.) [Our translation]

\section{THE EXPOSITION OF EVIL IN LORD OF THE FLIES AND PADMA NADIR MAJHI}

Evil is common in both of the novels. In Lord of the Flies, evil is exposed through the teenage boys. On the typical island, children are supposed to avoid ferocity to survive. But they show their merciless and savage nature with the progress of the story. The children who were the symbol of innocence, prove themselves blood-thirsty and irrational beings. The experience on the island is an end of innocence because the boys choose wildlife over rationality and awareness, "By choosing children to be the protagonists of his story, Golding emphasizes the theme that evil is inherent in man's nature and that childhood innocence serves only to veil it" (qtd. in Demir, 2019, p. 50). In the novel, Golding negates the traditional concept of an ideal state as to him, there is no ideal state in the human world. The evil resides everywhere.

On the other hand, in Padma Nadir Majhi, evil is revealed in two forms-carnal desire and societal exploitation. Sexual desire or biological need is a basic human desire and it is innate. But most of the leading characters of the novel try to satisfy their hunger in an unethical way. Influenced by their desire, Kuber makes an extramarital relationship with Kopila. The other characters are also influenced by this need either covertly or overtly. Hossain Mia plays his role in sexual activities. After coming back to Ketupur, the vitality in Rashu rises again. His sexual desire becomes uncontrollable and it makes him take an act of extreme revenge on Kuber. We also can identify the evil of the socio-economic system in the novel. Hossain Mia performs the role of one of the vehement exploiters who exploits the fishermen very covertly. Dhananjoy, Shital Ghosh, and Kedarnath are also the symbols of evils who oppress the fishermen of Ketupur in different ways. Dhananjoy takes more money and deprives Kuber and Ganesh of their rights. So, they are cheated on in every possible way by the ruling class people. 


\section{CONCLUSION}

Evil plays a significant role in Lord of the Flies and Padma Nadir Majhi. In Lord of the Flies, evil is presented through children, more particularly through Jack and his choir. The death of the noble-minded Simon and the wise Piggy occur due to the evil nature of Jack and his followers. Golding had personal experience of the two world wars and observed what wars did to human society. His philosophy was to restore principles in an unprincipled world. With this philosophy in mind, he exposed the innate evil of man in Lord of the Flies. On the other hand, in Padma Nadir Majhi evil is presented in the forms of carnal desire and societal exploitation. The characters fulfill their carnal desire in an unethical way and in this way, they commit evil deeds. Thus, the innate form of 'evil' is common in both novels. Except this, we find the evil created by the societal system in Padma Nadir Majhi. Thus, it can be said that no matter what a person's age, status, culture, or gender is, he/she possesses 'evil' in his/her character.

\section{REFERENCES}

[1] Bandapaddhaya, Manik. (1936). Padma Nadir Majhi. Dhaka, M. B. Printing and Publications.

[2] Demir, Ayse. (2019). "Violence Surpassing Innocence in Lord of the Flies by William Golding and The Bloody Chamber by Angela Carter". The Literary Trek, 5(1), pp.49 59.

https://www.researchgate.net/publication/334084647_Ayse _DEMIR_Violence _Surpassing_Innocence in L Lord of_The_Flies_by_William_Golding_and_The_Bloody Chamber by Angela Carter - Literacy Trek 20191

[3] Golding, William. (1954).Lord of the Flies. London, Faber and Faber.

[4] Hossain. Md. Amir. (2018). "Social Picture and Livelihood of the Boatmen in Manik Bandopadhyay's Padma Nadir Majhi: An Ecocritical Study". International Journal of Social Science Studies (IJSSS), 6(6), pp. 16-26.

https://www.researchgate.net/publication/325250494_Socia 1_Picture_and_Livelihood_of the_Boatmen_in_Manik_Ba ndopadhyay\%27s Padma Nadir Majhi An Ecocritical St udy

[5] Shakespeare, William. (1606). The Tragedy of Macbeth. London, Faber and Faber. 